\title{
An Offline System for the Recognition of the Fragmented Handwritten Numeric Chains
}

\author{
S. Ouchtati, M. Redjimi, and M. Bedda
}

\begin{abstract}
In this study we propose an off line system for the recognition of the fragmented handwritten numeric chains. Firstly, we realized a recognition system of the isolated handwritten digits, in this part; the study is based mainly on the evaluation of neural network performances, trained with the gradient back propagation algorithm. The used parameters to form the input vector of the neural network are extracted on the binary images of the handwritten isolated digit by several methods: the distribution sequence, sondes application, the Barr features, and the centered moments of the different projections and profiles. Secondly, the study is extended for the reading of the fragmented handwritten numeric chains constituted of a variable number of digits. Vertical projection was used to segment the numeric chain at isolated digits and every digit (or segment) was presented separately to the entry of the system achieved in the first part (recognition system of the isolated handwritten digits).
\end{abstract}

Index Terms-Handwritten numeric chains, optical characters recognition, neural networks, barr features, image processing, pattern recognition, features extraction.

\section{INTRODUCTION}

Handwritten numerical string recognition has been a topic of intensive research in recent years due to its large number of potential applications. The recognition of numerical strings differs from that of isolated digits because it requires the segmentation of a string into separate entities representing individuals digits. It is also deferent from the problem of recognizing handwritten words form a dictionary in the sense that almost no contextual information is available. Segmentation of numerical strings is generally a difficult task because individual numerals in a string can overlap or touch each other, or a numeral can be broken into several parts. Strategies for handwritten numerical string recognition can be divided into segmentation-then-recognition [1], [2] and segmentation-based recognition [3], [4]. In the first approach, the segmentation module provides a single sequence hypothesis where each sub-sequence should contain an isolated character, which is submitted to the recognizer. Very often, contextual information is used in the segmentation process to improve the robustness of the system. The second

Manuscript received August 10, 2014; revised November 12, 2014. This work was supported in part by the Electronic Research Laboratory of Skikda, University of August 20, 1955, Algeria and Electrical Engineering Department, Al-jouf University - Arabie Saoudite.

Salim Ouchtati is with the Electronic Research Laboratory of Skikda, University of August 20, 1955, Route El Hadaik, Bp: 26 Skikda 21000, Algeria (e-mail: ouchtatisalim@ yahoo.fr).

Mohammed Rdjimi is with Computer Science Department Electronics Department, University of August 20, 1955, Route El Hadaik, Bp: 26 Skikda 21000, Algeria (e-mail: medredjimi@gmail.com).

Mouldi Bedda is with the Electrical Engineering Department, Al-Jouf University, Arabie Saoudite, Algeria (e-mail: mouldi_bedda@ yahoo.fr). strategy is based on a probabilistic assumption where the final decision must express the best segmentation-recognition score of the input image. Usually, the system yields a list of hypotheses from the segmentation module and each hypothesis is then evaluated by the recognition. Finally, the list is post-processed taking into account the contextual information. Although this approach gives a better reliability than the previous one, the main drawback lies in the computational effort needed to compare all the hypotheses generated. Moreover, the recognition module has to discriminate various configurations such as fragments, isolated characters and connected characters. In this strategy, segmentation can be explicit when based on cut rules [5], [6] or implicit when each pixel column is a potential cut location [7], [8]. A good review about segmentation can be found in [9]. Indeed, handwritten numerical string recognition is present in almost every application involving handwriting recognition, for instance, postal address [10], [11], cheque processing and form reading [3], [7]. Such applications have been very popular in handwriting recognition research, due to the possibility to reduce considerably the manual effort involved in these tasks.

\section{A RECOGNITION SYSTEM FOR THE HANDWRITTEN ISOLATED DIGITS}

In the setting of the handwritten writing recognition, we proposed an off line system for the recognition of the isolated handwritten digits, this system is divided in three phases:

- Acquisition and preprocessing.

- Features extraction.

- Recognition.

A. Acquisition and Preprocessing

1) Acquisition

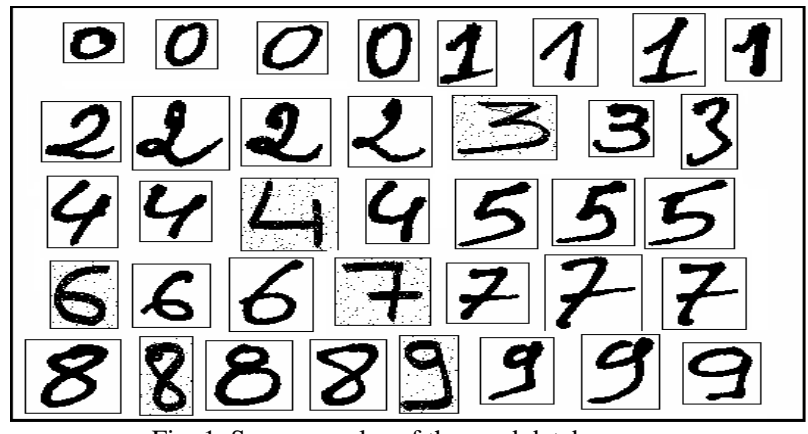

Fig. 1. Some samples of the used database.

Before analyzing the different processing steps, let's recall that we are especially interested at the off line processing. For our case, the acquisition is done with a numeric scanner of resolution 300 dpi with 8 bits/pixels, the used samples are all 
possible classes of the handwritten digits $(0,1,2,3,4,5,6,7$, $8,9)$ with variable sizes and variable thickness, and with and with one thousand (1000) samples for every class. Let's note that the characters images of our database are formed only by two gray levels: the black for the object and the white for the bottom. The Fig. 1 shows some samples of the used database.

\section{2) Preprocessing}

The preprocessing operations are classical operations in image processing, their objective is to clean and prepare the image for the other steps of the system. The preprocessing attempts to eliminate some variability related to the writing process and that are not very significant under the point of view of the recognition, such as the variability due to the writing environment, writing style, acquisition and digitizing of image. For our case, we used the following preprocessing operations:

\section{a) Filtering and inversion of the gray levels}

This operation consists in eliminating the noises in the binary image due to different reasons (bad Acquisition conditions , bad writing conditions, the writer's mood.. etc.), in our case, some digits are marked by the noise of type "peppers and salt", the application of the filter median on the digit image permitted us to eliminate easily this type of noise. Let's note that for reasons of calculation we reversed the gray levels of the character image (black for the bottom and white for the object). The Fig. 2 shows us the filtering and inversion operation of the gray levels of some handwritten digits.

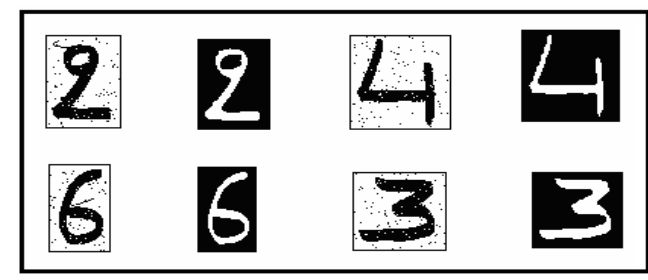

Fig. 2. Filtering and inversion of the gray levels of some handwritten digits.

\section{b) Normalization of the digit image}

Knowing that the digits images have variable sizes, this operation consists at normalizing the image size at $64 \times 64$ pixels (see Fig. 3).

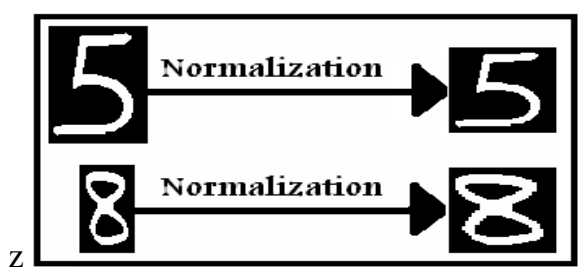

Fig. 3. Normalization of some handwritten digits.

\section{B. Features Extraction}

Features extraction is an important step in achieving good performance of OCR systems. However, the other steps also need to be optimized to obtain the best possible performance, and these steps are not independent. The choice of features extraction method limits or dictates the nature and output of the preprocessing step and the decision to use gray-scale versus binary image, filled representation or contour, thinned skeletons versus full-stroke images depends on the nature of the features to be extracted. Features extraction has been a topic of intensive research and we can find a large number of features extraction methods in the literature [12], but the real problem for a given application, is not only to find different features extraction methods but which features extraction method is the best ?. This question led us to characterize the available features extraction methods, so that the most promising methods could be sorted out. In this paper, we are especially interested in the binary image of the digits, and the methods used to extract the discrimination features of are the following:

\section{1) The distribution sequence}

This method has been used with success in several works [13], [14], while dividing the digit image at a determined number of zones, the distribution sequence characterizes a number of the object pixels in relation to the total pixels number in a given zone. For our application, the digit image is divided in 64 zones (see Fig. 4), and every zone is of size $8 \times 8$ pixel and the values of the distribution sequence are defined by:

$$
x_{i}=\frac{N_{i}}{N} \text { ? }
$$

With:

$x_{i}$ : is the ith value of the distribution sequence.

$N i:$ is a number of the object pixels in the ith zone.

$N$ : is a total pixels number in the ith zone.

\section{2) Application of the sondes}

This method is based on measuring the normalized distance between the border of the image and the first object pixel encountered (see Fig. 5), from this point of view, we can define the following probes distance:

- Top vertical Sondes

- Low vertical Sondes

- Left horizontal Sondes

- Right horizontal Sondes

Let's note that each sonde is applied every eight pixels which allows us to encode the digit image with thirty two (32) parameters.

\section{3) Barr-features}

The Barr features have been used with success in several works [14], [15], they are calculated on the binary digits images. Firstly, four images parameters are generated, and every image parameter corresponds to one of the following directions: east (e), North (n), Northeast (ne), Northwest ( $n w)$. Every image parameter has a whole value representing the Barr length in the direction in question. The features are calculated from the images parameters using zones that overlap to assure a certain degree of smoothing. Fifteen rectangular zones are arranged in five lines with three zones for every line; every zone is of size $[(\mathrm{h} / 3) \times(\mathrm{w} / 2)]$ where $\mathrm{h}$ and $\mathrm{w}$ are respectively the height and the width of the image. The high corners on the left of the zones are at the positions $\left\{\left(r_{0}\right.\right.$, $\left.c_{0}\right): r_{0}=0, \mathrm{~h} / 6,2 \mathrm{~h} / 6,3 \mathrm{~h} / 6,4 \mathrm{~h} / 6$ and $\left.c_{0}=0, \mathrm{w} / 4,2 \mathrm{w} / 4\right\}$. The values in every zone of the parameters images are added and the sums are normalized, and the dimension of the features vector is $15 \times 4=60$. If we suppose $f_{1}, f_{2}, f_{3}, f_{4}$ are the images parameters associated at a shape in entry and $Z i(i=1,2 \ldots .15)$ is an rectangular zone of size $[(\mathrm{h} / 3) \times(\mathrm{w} / 2)]$ with the top corner on the left is $\left(r_{0}, c_{0}\right)$, the value $P_{i k}$ of the parameter 
associated to the $Z i$ zone for the image parameter $f_{k}(k=1,2,3$, 4) is given like follows:

$$
P_{i k}=\frac{1}{N} \sum_{r=r_{0}}^{r_{0}+\frac{w}{2}} \sum_{c=c 0}^{c 0+\frac{h}{3}} f_{k}(r, c)
$$

With $N$ is a factor of normalization (for our application $N=64$ ).

The Fig. 6 shows the images parameters of the digits three and its Barr features.

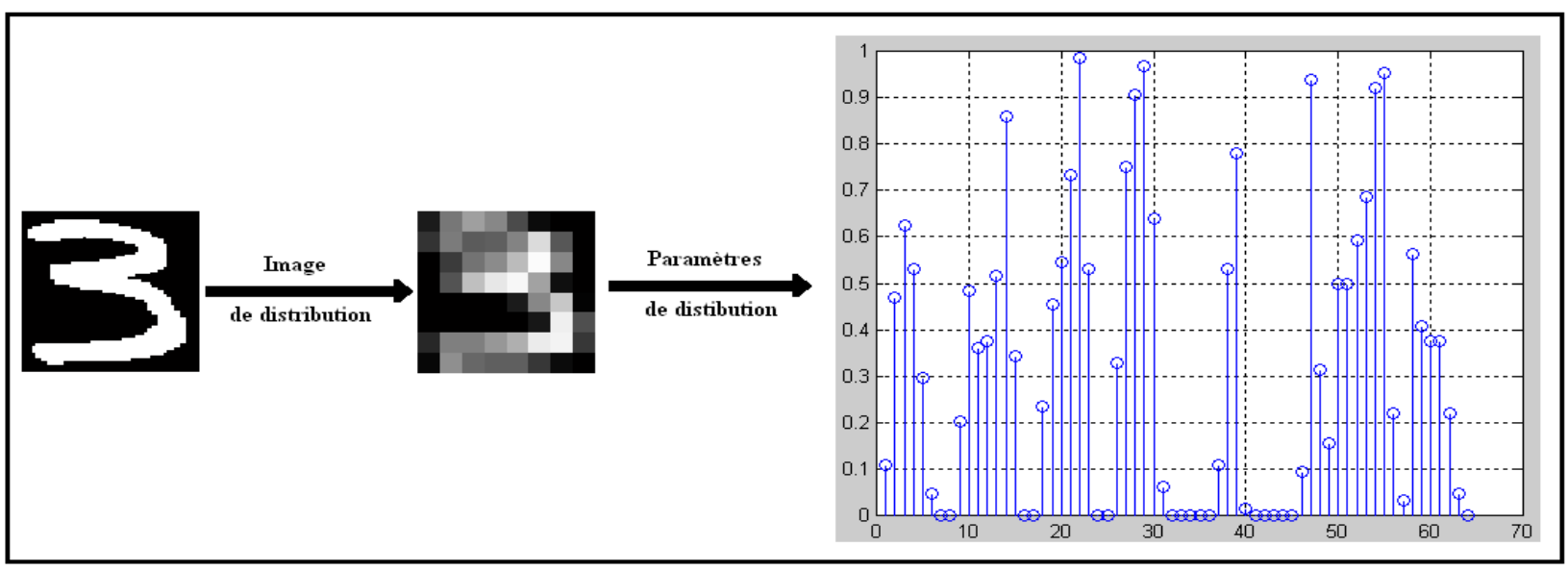

Fig. 4. The digits three and its distribution sequences.

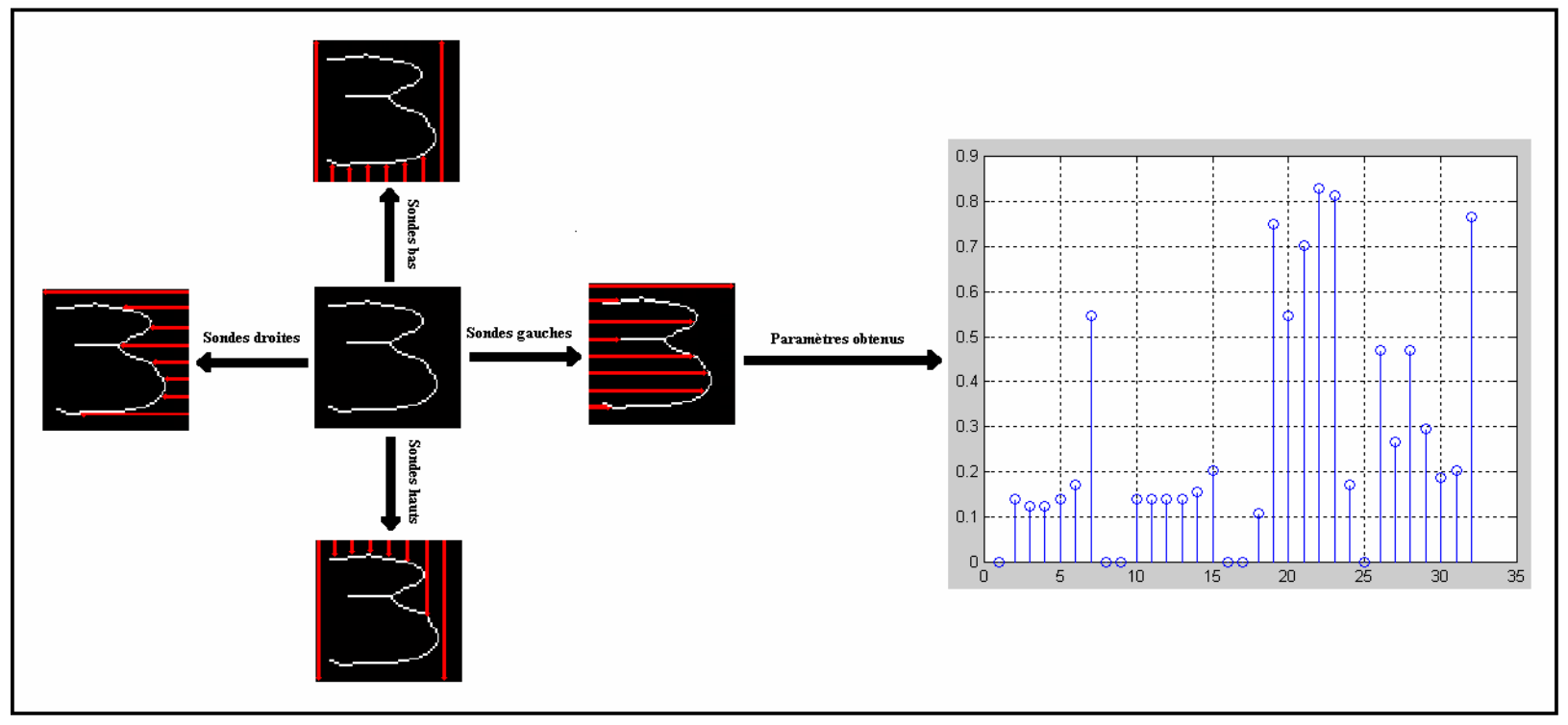

Fig. 5. Application of the sondes on the handwritten digit three.

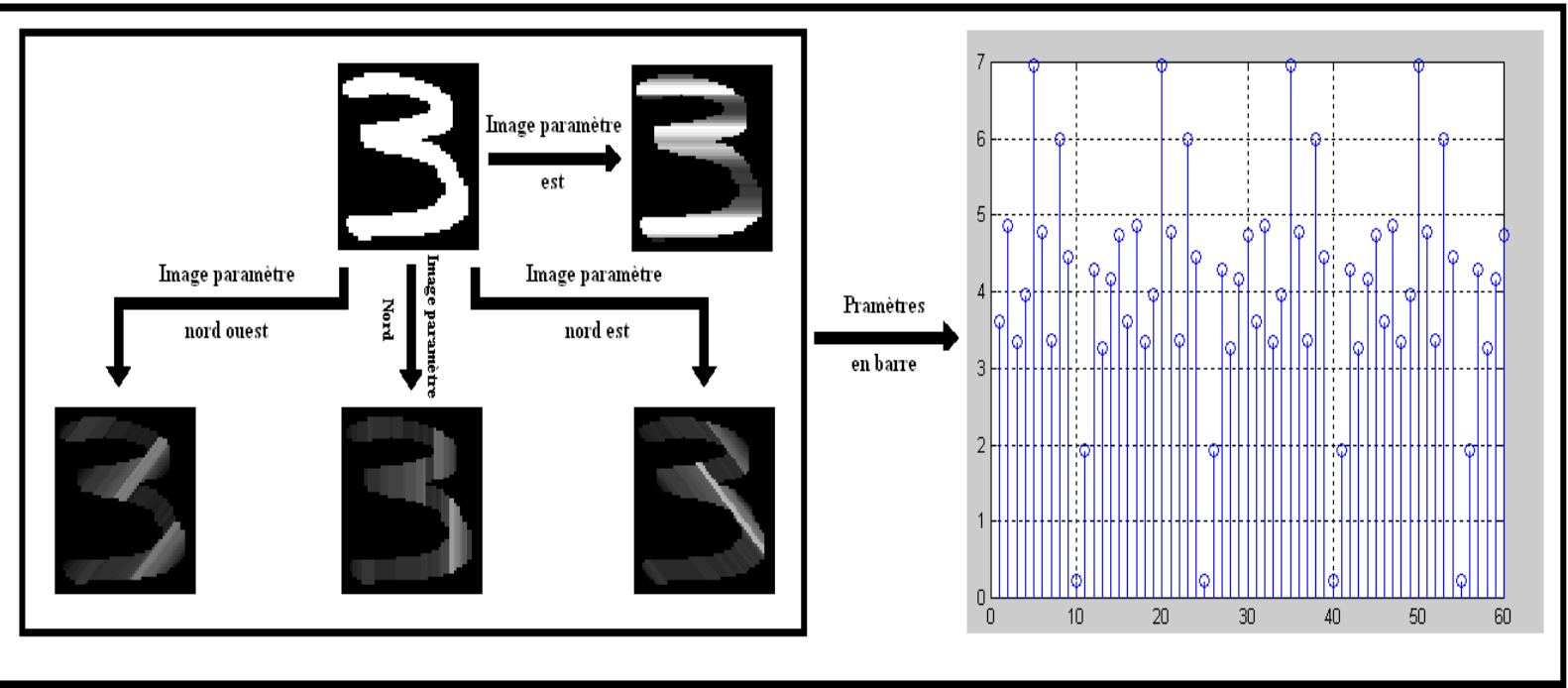

Fig. 6. The Images parameters of the digit three and its barr features. 


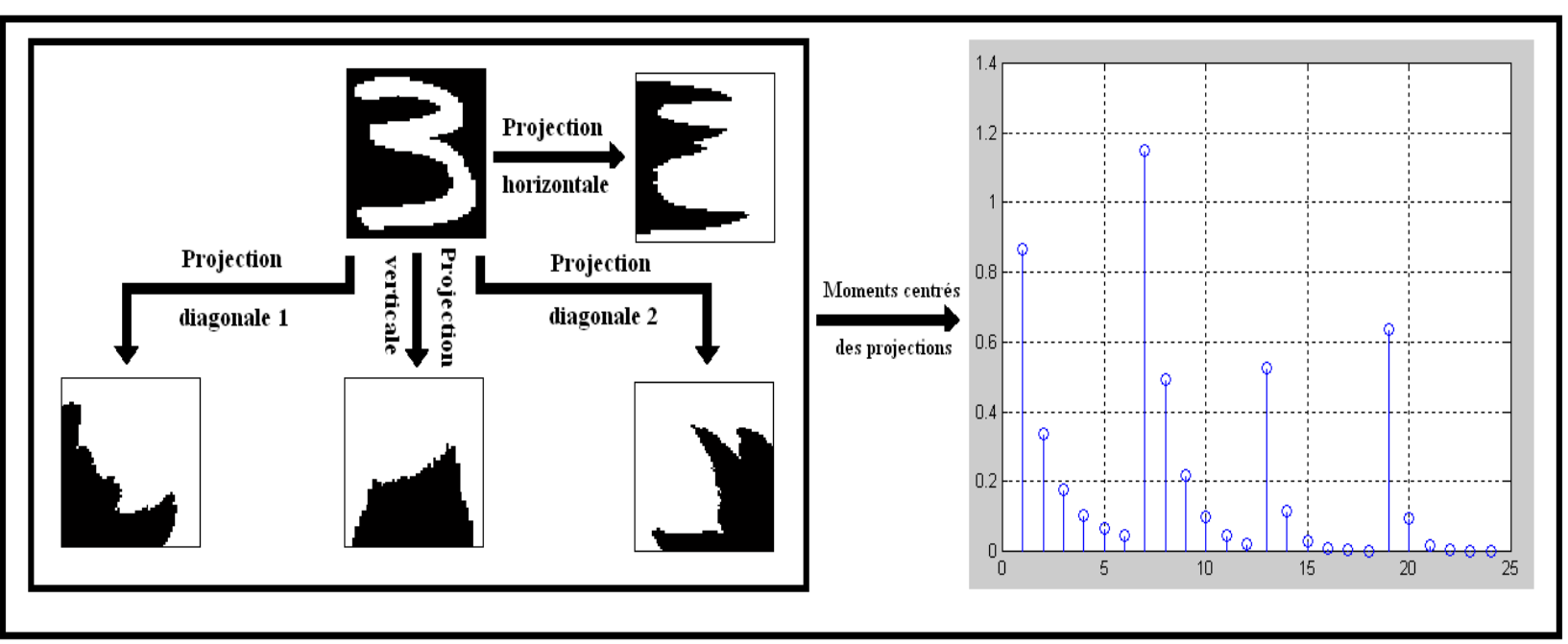

Fig. 7. The digits three and its different projections.
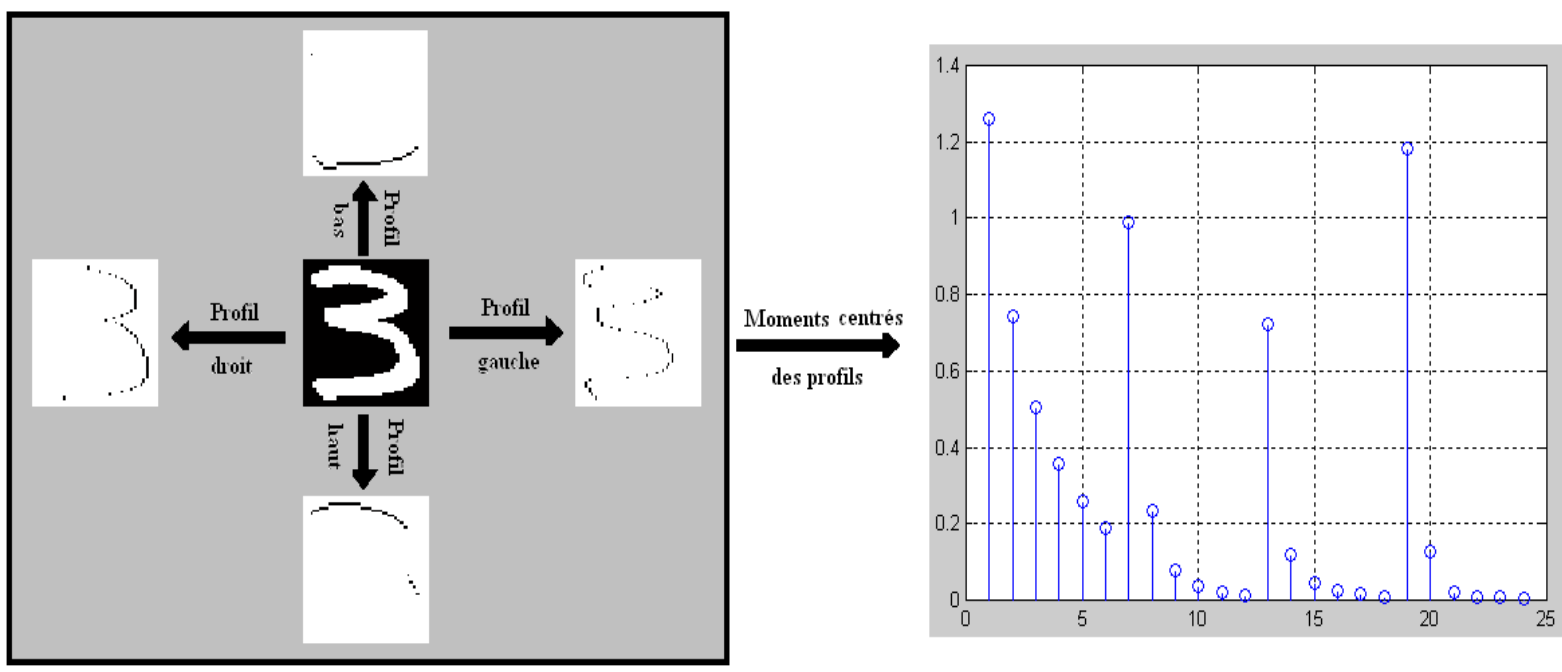

Fig. 8. The digits three and its different profiles

\section{4) The centered moments of the different projections.}

This method have been used with success in several works [16], In this case, the discrimination parameters are the centered moments extract from the follow projections (see Fig. 7):

- The vertical projection: for a given column, the value of the projection is equal to the number of object pixels in this column.

- The horizontal projection: for a given line, the value of the projection is equal to the number of object pixels in this line.

- The diagonals projections: for a given diagonal, the value of the projection is equal to the number of object pixels according the direction in question.

While the centered moments of the projection are given by the following formulas:

$$
\begin{gathered}
u_{k}=\sum_{i=1}^{M}\left(x_{i}-\bar{x}\right)^{k} \cdot p\left(x_{i}\right) \\
p\left(x_{i}\right)=\operatorname{prob}\left[x=x_{i}\right]
\end{gathered}
$$

$$
\bar{x}=\sum_{i=1} x_{i} p\left(x_{i}\right)
$$

For our application, we chose the first six moments for every projection.

\section{5) The centered moments of the different profiles}

By definition, the image profile in a given direction is the set of the object pixels seen while standing in this direction. From this point of view, we can define the following profiles (see Fig. 8):

- Low profile: it is the set of the object pixels seen while standing below the image of the number

- High profile: it is the set of the object pixels seen while standing over the image of the number

- Left profile: it is the set of the object pixels seen while standing on the left of the image of the number

- Right profile: it is the set of the object pixels seen while standing on the right of the image of the number

While the centered moments of the different profiles are calculated by formulas: (3), (4) and (5).

For our application, we chose the first six moments for every profile. 


\section{6) Used features vector}

It is the features vector used to characterize the word image, and with which, we will nourish the recognition module. For our case the vector used is formed by the sixty four (64) parameter obtained by the distribution sequence method, thirty two (32) parameter obtained by the application of the sondes, the sixty (60) parameter obtained by the Barr-features method, the twenty four (24) parameter obtained by the centered moments of the different projections and the twenty four (24) parameter obtained by the centered moments of the different profiles.

\section{Digit Recognition}

The handwritten digits recognition is a problem for which a recognition model must necessarily take in account an important number of variabilities, dice at the time, the recognition techniques based on the neural networks can bring certain suppleness for the construction of such models. For our system, we opted for an MLP (Multi-Layers Perceptron) which is the most widely studied and used neural network classier. Moreover, MLPs are efficient tools for learning large databases. The used MLP in our work is trained with the back propagation with momentum training algorithm. The transfer function employed is the familiar sigmoid function.

\section{1) The input data}

The database consists of ten thousand (10000) binary images. These images represent all classes possible of the handwritten digits $(0,1,2,3,4,5,6,7,8,9)$ with variable sizes and variable thickness, and with one thousand (1000) samples for every class. This database is divided to two sets, $70 \%$ for training the neural network and $30 \%$ for testing it

\section{2) Neural network parameters}

The structure of the used neural network vary depending of the used vector parameters, the input layer nodes number is equal to the size of the used features vector $($ N_IL=204), the output layer nodes number is equal to the classes number to recognize $\left(\mathrm{N} \_\mathrm{OL}=10\right)$, for the hidden layers, we used a double hidden layer, and the number of nodes for each hidden layer is fixed by groping ( for our case N_HL1=107 and N_HL2=45) The initial connection weights are in the range $[-1,1]$.

\section{3) The training process}

For training the neural network, back propagation with momentum training method is followed. This method was selected because of its simplicity and because it has been previously used on a number of pattern recognition problems. The method works on the principle of gradient descent. The algorithm uses two parameters which are experimentally set, the learning rate $\eta$ and momentum $\mu$. These parameters allow the algorithm to converge more easily if they are properly set by the experimenter. For our case, we have opted for the following values: $\eta=0.35$ and $\mu=0.9$. During the learning phase the neural network learns by example and the connection weights are updated in an iterative manner. The training process for the network is stopped only when the sum of squared error falls below 0.001 .

4) The experimental results

The neural network performances are measured on the entire database (training or learning set and testing set). During this phase, we present the digit image to recognize to the system entry, and we collect at the exit its affectation to one of the possible classes.

The results can be:

- Recognized digit: the system arrives to associate one and only one prototype to the digit to recognize.

- Ambiguous digit: the system proposes several prototypes to the digit to recognize.

- Rejected digit: the system doesn't take any decision of classification.

- Non recognized digit: the system arrives to take a decision for the presented digit, but it is not the good decision.

The results and the different rates are regrouped in the Table I:

TABLE I: RESULTS AND DIFFERENT OBTAINED RATES

\begin{tabular}{|c|c|c|c|}
\hline $\mathbf{R} \_\mathbf{R}(\%)$ & A_R(\%) & J_R(\%) & UR_R(\%) \\
\hline 97.69 & $\mathbf{1 . 2 3}$ & $\mathbf{0 . 8 5}$ & $\mathbf{0 . 2 3}$ \\
\hline
\end{tabular}

\section{FRAGMENTED HANDWRITTEN NUMERIC CHAINS RECOGNITION}

The fragmented handwritten numeric chains are chains that contain at least one fragmented digit (see Fig. 9). In this part, we tried to widen the system realized in the part one of this paper, not only to the recognition of the isolated handwritten digits, but also to the recognition of the fragmented handwritten numeric chains, the used database contains one thousand (1000) fragmented handwritten numeric chains, constituted of a variable number of digits and with different lengths and different writers. The acquisition of this database is done with a numeric scanner of resolution 300 dpi with 8 bits/pixels.

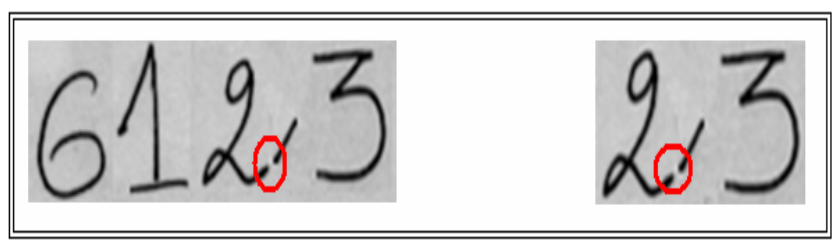

Fig. 9. Example of some fragmented habdwritten numeric chains.

\section{A. Segmentation of the Fragmented Handwritten Numeric Chains}

The new system and after presentation of the fragmented handwritten numeric chain, begins by binarizing the image of the numeric chain (see Fig. 10), then determine its vertical projection (see Fig. 11) on which is applied a procedure improvements to eliminate the effects caused by fragmented digits (the digits fragments are considered as isolated digits). The improvement process is to consider in the vertical image projection, all space upper than a definite threshold (for our case we have chosen the threshold $=2$ pixels) as an area of segmentation. Otherwise (the space is less than the threshold), the space is replaced by objects pixels and the effects of fragmented digits are avoided (see Fig. 12), and finally, The segmentation of the fragmented numeric chain in isolated digits is achieved by exploiting the transition black/white (or 
white/black) when we sweep for the last line the image columns of the vertical projection.

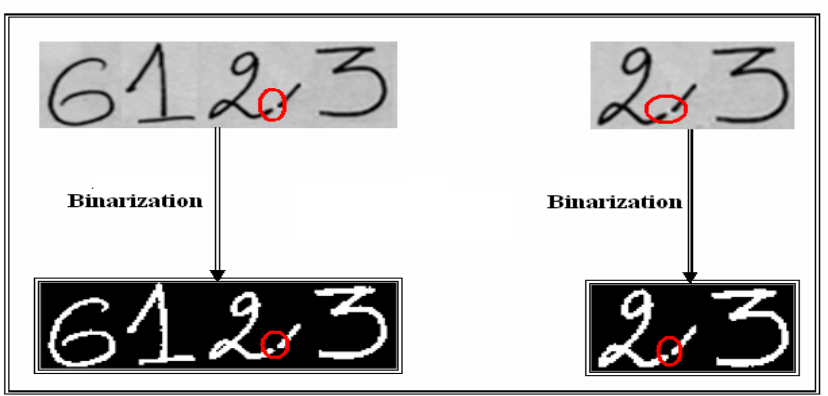

Fig. 10. Binarization of some fragmented handwritten numeric chains.

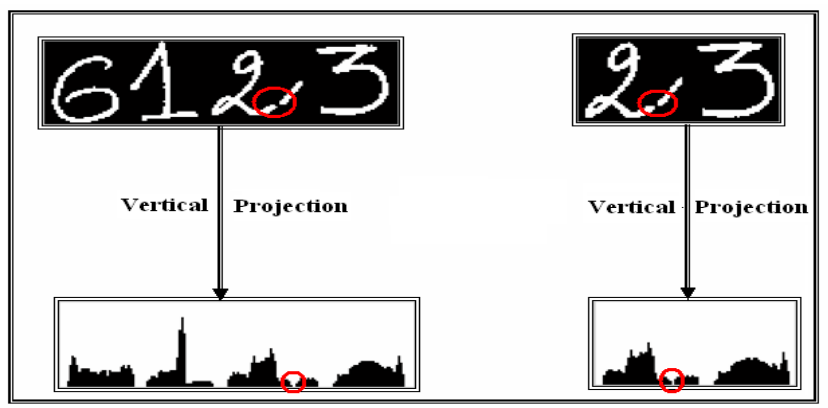

Fig. 11. Vertical Projection of some fragmented handwritten chains.

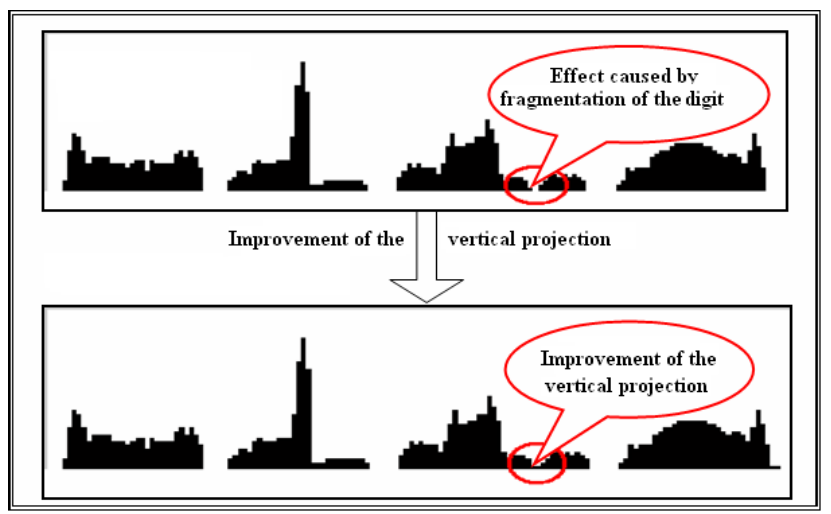

Fig. 12. Example of the improving of an fragmented numeric chain.

\section{B. Recognition of the Numeric Chain}

Every obtained digit by segmentation will be presented separately to the entry of the system achieved in the first part (recognition system of the isolated handwritten digits) and will undergo the following processing: normalization, features extraction and finally the recognition. Finally and after the presentation of all segments, the system displays the result of the recognition of the presented handwritten numeric chain, it may be:

- Recognized chain: In this case the system arrives to take the right decision about the chain presented at its enter.

- Rejected Chain: In this case the system cannot make a decision for at least one digit of the chain presented at its enter.

- Ambiguous Chain: In this case the system takes several decisions with the same degree confidence for at least one digit of the chain presented at its enter

- Unrecognized chain: In this case the system assigns at least one digit of the chain presented at its enter to a wrong answer.

The results and the different rates of this system part are regrouped in the Table II.

With:

- R-R: Recognizer rate A-R: Ambiguity rate.

- J-R: Reject rate. UR-R: Unrecognized rate.

C. Conclusion and Perspectives

TABLE II: RESULTS AND DIFFERENT OBTAINED RATES

\begin{tabular}{|c|c|c|c|}
\hline $\mathrm{R} \_\mathrm{R}(\%)$ & A_R(\%) & J_R(\%) & UR_R(\%) \\
\hline 96.3 & 1.70 & 1.20 & 0.80 \\
\hline
\end{tabular}

In our work, we presented an off line system for the recognition of the handwritten numeric chains. This work is divided in two big parts:

- The realization of a system of isolated digits recognition: in this case, the study is based mainly on the evaluation of neural network performances, trained with the gradient back propagation algorithm. The used parameters to form the input vector of the neural network are extracted on the binary images of the handwritten isolated digit by several methods: the distribution sequence, sondes application, the Barr features, and the centered moments of the different projections and profiles.

- The extension of our system for the reading of the fragmented handwritten numeric chains (constituted of a variable number of digits) by using the vertical projection to segment the numeric chain at the isolated digits and every digit will be presented separately to the entry of the system achieved in the first part (recognition system of the isolated handwritten digits). The obtained results are very encouraging and promoters; however we foresee to improve the realized system by taking in account the other segmentation problems and especially the problem of the overlapping digits and the attached digits in the numeric chain.

\section{REFERENCES}

[1] J. Allebach, "Binary display of images when spot size exceeds step size," Applied Optics, vol. 15, pp. 2513-2519, August 1980.

[2] E. Catmull, "A tutorial on compensation tables," Computer Graphics, vol. 13, pp. 1-7, 1979.

[3] P. Litwinowicz and L. Williams, "Animating images with drawings," in Proc. the Annual Conference Series, pp. 409-412, 1994.

[4] J. S. Bridle, "Probabilistic interpretation of feed forward classification network outputs, with relationships to statistical pattern recognition," Neurocomputing-Algorithms, Architectures and Applications, pp. 227-236, 1989.

[5] W. K. Chen, Linear Networks and Systems, Wadsworth, pp. 123-135, 1993.

[6] H. Poor. (1986). A Hypertext History of Multiuser Dimensions. [Online].

http://www.ccs.neu.edu/home/pb/mud-history.html

[7] K. Elissa, An Overview of Decision Theory, 1988.

[8] R. Nicole, "The last word on decision theory," Journal Computer Vision, submitted for publication.

[9] C. J. Kaufman, Rocky Mountain Research Laboratories, Boulder, Colo., personal communication, 1992.

[10] Y. Yorozu, M. Hirano, K. Oka, and Y. Tagawa, "Electron spectroscopy studies on magneto-optical media and plastic substrate interface," IEEE Trans. Magnetics, vol. 2, pp. 740-741, Aug. 1987.

[11] S. P. Bingulac, "On the compatibility of adaptive controllers," in Proc. the Fourth Ann. Allerton Conf. Circuits and Systems Theory, pp. 8-16, 1994. 
[12] J. M. Queen, "Some methods for classification analysis of multivariate observations," in Proc. the Fifth Berkeley Symp. Math. Statistics and Probability, pp. 281-297, 1967.

[13] S. Ouchtati, M. Bedda, and A. Lachouri "Segmentation and recognition of handwritten numeric chains," Journal of Computer Science, vol. 3, no. 4, 2007, pp. 242-248.

[14] S. Ouchtati, L. Bennacer, and M. Bedda, "An off line system for the arabic handwritten words recognition," International Review on Computers and Software, vol. 3, no. 6, 2008, pp. 579-585.

[15] S. Ouchtati and M. Bedda, "Multilayer neural network for the recognition of the Arabic handwritten words of the Algerian Department," in Proc. the International Conference on Applied Analysis and Mathematical Modeling, Yildiz Technical University, Istanbul, Terkey, 2013, pp. 1-5.

[16] S. Ouchtati, M. Redjimi, and M. Bedda, "Realization of an offline system for the recognition of the handwritten Numerci chains," in Proc the 9th Iberian Conference on Information Systems and Technologies, Barcelone Spain, 2014, pp. 749-754.

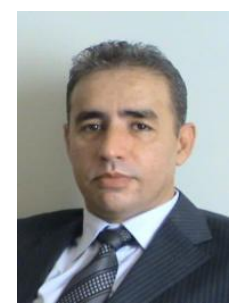

Salim Ouchtati was born in Azzaba W Skikda, Algeria in 1970. He received his B.Eng. and M.Sc. degrees in electronic from Annaba University, Algeria in 1994 and 1999. Respectively. He received his doctorate diploma in automatic in 2007. his received his HDR diploma (habilitation to direct the research) in electronic from Annaba University in 2010

$\mathrm{He}$ is currently an associate professor at Skikda university, a member of the Skikda Electronic Laboratory, responsible course of Industrial Control. His research interests include handwritten recognition, artificial intelligence and image processing.

Dr. Salim Ouchtati was a member of the scientific committee of the Electrical Engineering Department in Skikda University from 2005 to 2010, he was a member of the scientific committee of the Technology Faculty in the Skikda University from 2005 to 2008. He was a reviewer in several international conferences.

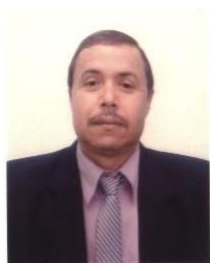

Mohammed Redjimi was born in Rejattas, Skikda, Algeria in 1956. he received his diploma of docteur ingenieur in computer science from the University of Lille 1, France in 1984 and He received his HDR diploma (habilitation to direct the research) in computer science from Annaba University in 2007.

$\mathrm{He}$ is currently an associate professor of computer science at Skikda University, responsible of the team research "Modeling and simulation of complex processes" at Skikda Computer Science and Communication Laboratory, responsible of the research project with the title: "Cooperative approaches for images segmentation," responsible of the research project with the title: "Bayesian approach in computer vision," he has a several scientific works published in many international journals. His main research interests include modeling and simulation systems mainly by using multi-agents systems, image recognition and computing hardware and software systems.

Dr Mohammed Redjimi was a reviewer and member of the scientific committee in several international conferences.

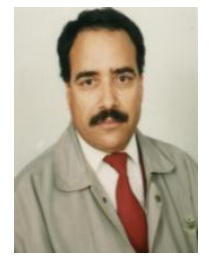

Mouldi Bedda was born in El_Oued Algeria in 1956. He received his bachelor's degree from the University of Haouari Boumedienne Algers, Algeria in 1981, He received his M.Sc degree from the university of Languedoc Montpelier French in 1982, and He received his Phd degree in electrical engineering from the university Nancy 2, Nancy, French in 1985.

He was a assistant professor from 1985-1990, he as a associate professor from 1990-2004 and he as a professor from 2004-2006 at the university of Annaba Algeria, from 2006 to date full professor at the college of engineering at aljouf university KSA. He has a several scientific works published in many international.

Professor Mouldi Bedda was the director of automatic and signals laboratory from 2001-2006. His researches interests include DSP, speech processing, OCR, artificial intelligence, and biomedical. 